PROCEEDINGS OF THE

AMERICAN MATHEMATICAL SOCIETY

Volume 126, Number 4, April 1998, Pages 973-982

S 0002-9939(98)04242-7

\title{
REMARKS ON NUMERICAL RANGES OF OPERATORS IN SPACES WITH AN INDEFINITE METRIC
}

\author{
CHI-KWONG LI AND LEIBA RODMAN
}

(Communicated by Palle E. T. Jorgensen)

\begin{abstract}
The numerical range of an operator on an indefinite inner product space (possibly infinite dimensional) is studied. In particular, operators having bounded numerical ranges are characterized, and the angle points of the numerical range and their connections with eigenvalues are described.
\end{abstract}

\section{INTRODUCTION}

Let $H$ be a (complex) Hilbert space, with the scalar product $(\cdot, \cdot)$, and let $S$ be a bounded self-adjoint operator on $H$. For a linear bounded operator $A: H \rightarrow H$ we define

$$
W_{S}^{ \pm}(A)=\left\{\frac{(S A x, x)}{(S x, x)}: x \in H,(S x, x)= \pm 1\right\} .
$$

For future reference define also $W_{S}^{0}(A)=\{(S A x, x): x \in H,(S x, x)=0\}$. The sets $W_{S}^{ \pm}(A)$ generalize the well-known and widely used notion of numerical range

$$
W(A)=\{(A x, x): x \in H,(x, x)=1\}
$$

of $A$. In fact, if $S$ is positive definite and invertible, then $W_{S}^{+}(A)=W\left(X A X^{-1}\right)$, where $X$ is any operator on $H$ such that $S=X^{*} X$. If $S$ is invertible but indefinite, then the sets $W_{S}^{ \pm}(A)$ can be understood as natural generalizations of the numerical range with respect to the Krein space structure defined by the indefinite scalar product $[x, y]=(S x, y), x, y \in H$.

The sets $W_{S}^{ \pm}(A)$, as well as other related sets, have been studied in [B, GP] (with the emphasis on the convexity properties) and in [LTU], where a more detailed description of the geometric shapes of $W_{S}^{ \pm}(A)$ is given. In this paper we characterize operators $A$ whose numerical range is bounded, and investigate the geometric properties of $W_{S}^{ \pm}(A)$ in relation to the spectrum of $A$. Some of our results are given for the finite-dimensional spaces $H$, in which case we identify $H$ with $\mathbf{C}^{n}$. Because $W_{S}^{-}(A)=W_{-S}^{+}(A)$, we will state and prove our results for $W_{S}^{+}(A)$ only, with the understanding that analogous results are valid for $W_{S}^{-}(A)$ as well.

We denote by $L(H)$ the algebra of all bounded linear operators on $H$. For selfadjoint operators $X, Y \in L(H)$ the notation $X \geq Y$ means that the difference

Received by the editors September 23, 1996.

1991 Mathematics Subject Classification. Primary 15A60, 47A12, 47A37.

Key words and phrases. Numerical range, indefinite scalar product, Krein space.

Research of both authors was partially supported by NSF Grants.

(C)1998 American Mathematical Society 
$X-Y$ is positive semidefinite. Throughout the paper it will be assumed that $H$ is separable.

\section{BASIC PROPERTIES}

We start by recalling some basic and known results.

The joint numerical range $W\left(G_{1}, \ldots, G_{k}\right)$ of $k$ self-adjoint operators $G_{1}, \ldots, G_{k}$ $\in L(H)$ on $H$ is defined by

$$
W\left(G_{1}, \ldots, G_{k}\right)=\left\{\left(\left(G_{1} x, x\right), \ldots,\left(G_{k} x, x\right)\right) \in \mathbf{R}^{k}: x \in H,(x, x)=1\right\} .
$$

This notion is a useful generalization of the classical numerical range, and is well studied (see, e.g., [AT, BL]). Let

$$
K\left(G_{1}, \ldots, G_{k}\right)=\bigcup_{\alpha \geq 0} \alpha W\left(G_{1}, \ldots, G_{k}\right)
$$

be the cone generated by $W\left(G_{1}, \ldots, G_{k}\right)$. Using the properties of the joint numerical range, the authors of [LTU] proved:

Proposition 2.1. Let $S=S^{*}, A \in L(H)$. If $G_{1}, G_{2}$ are self-adjoint operators such that $S A=G_{1}+i G_{2}$, then $K\left(G_{1}, G_{2}, S\right)$ is convex. Consequently,

$$
W_{S}^{+}(A)=\left\{x+i y \in \mathbf{C}:(x, y, 1) \in K\left(G_{1}, G_{2}, S\right)\right\},
$$

is also convex.

The next proposition is very useful in studying the geometric properties of $W_{S}^{+}(A)$.

Proposition 2.2. Let $S=S^{*}, A \in L(H)$.

(a) For every $\alpha, \lambda \in \mathbf{C}$, we have

$$
W_{S}^{+}(A+\lambda I)=W_{S}^{+}(A)+\lambda ; \quad W_{S}^{+}(\alpha A)=\alpha W_{S}^{+}(A) .
$$

(b) Let $H_{0} \subseteq H$ be a (closed) subspace in $H, P_{0}$ be the orthogonal projection on $H_{0}$, and let $P_{1}=I-P_{0}$. If $P_{0} S P_{1} \cdot P_{1} A P_{0}=0$. Then

$$
W_{P_{0} S \mid H_{0}}^{+}\left(P_{0} A \mid H_{0}\right) \subseteq W_{S}^{+}(A) .
$$

Proof. Part (a) is clear. To prove (b), let $x_{0} \in H_{0}$ be such that $\left(P_{0} S x_{0}, x_{0}\right)=1$. Then it is easy to see that $\left(S x_{0}, x_{0}\right)=\left(P_{0} S x_{0}, x_{0}\right)=1$. The condition $P_{0} S P_{1}$. $P_{1} A P_{0}=0$ guarantees that

$$
\left(P_{0} S P_{0} \cdot P_{0} A x_{0}, x_{0}\right)=\left(S A x_{0}, x_{0}\right) .
$$

So the typical element $\left(P_{0} S P_{0} \cdot P_{0} A x_{0}, x_{0}\right)$ of $W_{P_{0} S \mid H_{0}}^{+}\left(P_{0} A \mid H_{0}\right)$ also belongs to $W_{S}^{+}(A)$.

Besides convexity, another basic property for the classical numerical range is the boundedness. Actually, $W(A)$ is always a compact convex set in the finite dimensional case. However, as shown in [LTU, Section 2], $W_{S}^{+}(A)$ may be neither closed nor bounded in $\mathbf{C}$, even in the finite dimensional case. In fact, it was pointed out in Proposition 4.1 of [LTU] that if $S$ is indefinite and $S A \neq \lambda S$ for any $\lambda \in \mathbf{C}$, then $W_{S}(A)$ is unbounded. In the following, we give complete characterization for $S$ and $A$ such that $W_{S}(A)$ is nonempty and bounded.

Theorem 2.3. Let $S=S^{*}, A \in L(H)$. Then $W_{S}^{+}(A)$ is nonempty and bounded if and only if EITHER 
(a) $S$ is indefinite and $S A=\lambda S$, for some $\lambda \in \mathbf{C}, O R$

(b) the properties (i)-(ii) below are satisfied:

(i) $S \geq 0$ and $S \neq 0$;

(ii) the linear set $\operatorname{Ran} S^{\frac{1}{2}}$ is $A^{*}$-invariant, where $S^{\frac{1}{2}}$ is the positive semidefinite square root of $S$.

Proof. Clearly, if (a) holds, then $W_{S}^{+}(A)=\{\lambda\}$. Assume (i) and (ii) hold. By Douglas' lemma (see [D]), there exists $\lambda \geq 0$ such that $A^{*} S A \leq \lambda S$. Then for every $x \in H$ :

$$
\begin{aligned}
|(S A x, x)|^{2} & =\left|\left(S^{\frac{1}{2}} A x, S^{\frac{1}{2}} x\right)\right|^{2} \leq\left(S^{\frac{1}{2}} A x, S^{\frac{1}{2}} A x\right) \cdot\left(S^{\frac{1}{2}} x, S^{\frac{1}{2}} x\right) \\
& =\left(A^{*} S A x, x\right)(S x, x) \leq \lambda(S x, x)^{2} .
\end{aligned}
$$

Thus, $|z| \leq \lambda^{\frac{1}{2}}$ for every $z \in W_{S}^{+}(A)$, and hence $W_{S}^{+}(A)$ is bounded.

Conversely, assume that $W_{S}^{+}(A)$ is nonempty and bounded. Assume first that $S$ is indefinite. Let $H_{1} \subset H_{2} \subset \cdots$ be a sequence of finite-dimensional subspaces such that

$$
\overline{\bigcup_{m} H_{m}}=H
$$

and $P_{m} S P_{m} \in L\left(H_{m}\right)$ is indefinite (here $P_{m}$ is the orthogonal projection on $H_{m}$ ). Using Proposition 2.2(b) and applying the finite dimensional result (Theorem 2.4 of [LTU]) to $P_{m} S P_{m}$ and $P_{m} A P_{m}$, we obtain that

$$
(S A x, y)=(\lambda S x, y), \quad x, y \in H_{m},
$$

where the constant $\lambda$ is independent of $m$. Now the condition (2.1) guarantees that $S A=\lambda S$.

Next, assume that $W_{S}^{+}(A)$ is non-empty and bounded, but $S$ is positive semidefinite and nonzero. We will prove that (ii) holds. Suppose $y=A x \notin \operatorname{Ker} S$ for some $x \in \operatorname{Ker} S$. Let $z=S y \neq 0$. Clearly, $z \perp \operatorname{Ker} S$. For a positive number $\delta$ we have

$$
\begin{gathered}
(S(x+\delta z), x+\delta z)=\delta^{2}(S z, z) \\
(S A(x+\delta z), x+\delta z)=\delta(S A(x+\delta z), z)=\delta[(z, z)+\delta(S A z, z)] .
\end{gathered}
$$

Therefore, $W_{S}^{+}(A)$ contains

$$
\frac{(S A(x+\delta z), x+\delta z)}{(S(x+\delta z), x+\delta z)}=\delta^{-1} \frac{(z, z)}{(S z, z)}+\text { const. }
$$

which tends to infinity as $\delta$ tends to 0 , a contradiction with the boundedness of $W_{S}^{+}(A)$. Thus, Ker $S$ is $A$-invariant, or, equivalently, $\overline{\operatorname{Ran} S}=\overline{\operatorname{Ran} S^{\frac{1}{2}}}$ is $A^{*}$ invariant.

Let $S_{0}=P S P, A_{0}=P A P \in L(\overline{\operatorname{Ran} S})$, where $P$ is the orthogonal projection on $\overline{\operatorname{Ran} S}$. For $x \in H$, write $x=x_{1}+x_{0}$, where $x_{1} \in \operatorname{Ker} S, x_{2} \in \overline{\operatorname{Ran} S}$. Because Ker $S$ is $A$-invariant, it is easy to see that $(S A x, x)=\left(S_{0} A_{0} x_{0}, x_{0}\right) ;(S x, x)=\left(S_{0} x_{0}, x_{0}\right)$. So $W_{S}^{+}(A)=W_{S_{0}}^{+}\left(A_{0}\right)$. In other words, we can (and do) assume that Ker $S=\{0\}$. Define the linear operator $C: \operatorname{Ran} S^{\frac{1}{2}} \rightarrow \operatorname{Ran} S^{\frac{1}{2}}$ by $C S^{\frac{1}{2}} x=S^{\frac{1}{2}} A x, x \in H$. We 
then have, for $x \in H, x \neq 0$,

$$
\frac{(S A x, x)}{(S x, x)}=\frac{\left(S^{\frac{1}{2}} A x, S^{\frac{1}{2}} x\right)}{\left(S^{\frac{1}{2}} x, S^{\frac{1}{2}} x\right)}=\frac{(C y, y)}{(y, y)} \in W_{S}^{+}(A),
$$

where $y=S^{\frac{1}{2}} x$. Since $W_{S}^{+}(A)$ is bounded, we have $|(C y, y)| \leq M$ for all $y \in$ $\operatorname{Ran} S^{\frac{1}{2}},\|y\|=1$, where $M>0$ is independent of $y$. Using the polarization identity, we see that $|(C x, y)| \leq 4 M$ for all normalized vectors $x, y \in \operatorname{Ran} S^{\frac{1}{2}}$. Thus, $C$ is a bounded operator, and therefore can be extended to a bounded linear operator (also denoted by $C$ ) on $H$. Now we have $A^{*} S^{\frac{1}{2}}=S^{\frac{1}{2}} C^{*}$, and (ii) is obvious.

Note that (ii) can be stated in an equivalent form (see [D]):

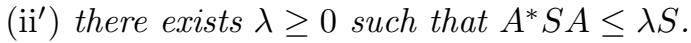

We also note that if $W_{S}^{+}(A) \neq \emptyset$ is bounded, then either $W_{S}^{+}(A)=\{\lambda\}$ (if $S$ is indefinite), or $S \geq 0, S \neq 0$ and

$$
W_{S}^{+}(A)=\left\{(C y, y): y \in \operatorname{Ran} S^{\frac{1}{2}},(y, y)=1\right\},
$$

for some $C \in L(\overline{\operatorname{Ran} S})$; in fact, $C$ is explicitly given by $C S^{\frac{1}{2}}=S^{\frac{1}{2}} A$.

It is well-known that if $H$ is an orthogonal sum of two $A$-invariant subspaces $H_{1} \oplus H_{2}$, and if $A$ is written in the form $A=A_{1} \oplus A_{2}$ with $A_{j} \in L\left(H_{j}\right)$ for $j=1,2$, then $W(A)=\operatorname{conv}\left\{W\left(A_{1}\right) \cup W\left(A_{2}\right)\right\}$ (we denote by $\operatorname{conv}\{X\}$ the convex hull of the set $X)$. An extension of this result to $W_{S}(A)$ is desirable. We will say that $H$ has a direct sum decomposition compatible with $S$ and $A$ if $H$ is a direct sum of two $A$-invariant (closed) subspaces: $H=H_{1} \oplus H_{2}$ such that $(S x, y)=0$ for all $x \in H_{1}, y \in H_{2}$. We shall write $A=A_{1} \oplus A_{2}$ and $S=S_{1} \oplus S_{2}$, with $A_{j}, S_{j}$ acting on $H_{j}$ for $j=1,2$, if such a decomposition exists. Of course, for each $j=1,2$, one may consider $W_{S_{j}}^{+}\left(A_{j}\right), W_{S_{j}}^{-}\left(A_{j}\right), W_{S_{j}}^{0}\left(A_{j}\right)$. By Proposition 2.2(b), we see that $W_{S_{j}}^{+}\left(A_{j}\right) \subseteq W_{S}(A)$. To give a complete description of $W_{S}(A)$ in terms of sets associated with $S_{j}$ and $A_{j}$ for $j=1,2$, we need to consider complex numbers of the forms:

(1) $z=\alpha z_{1}-(1-\alpha) z_{2}$ for some $\alpha>1, z_{1} \in W_{S_{1}}^{+}\left(A_{1}\right), z_{2} \in W_{S_{2}}^{-}\left(A_{2}\right)$;

(2) $z=z_{1}+z_{2}$ for some $z_{1} \in W_{S_{1}}^{+}\left(A_{1}\right), z_{2} \in W_{S_{2}}^{0}\left(A_{2}\right)$;

(3) $z=\alpha z_{1}+(1-\alpha) z_{2}$ for some $1>\alpha>0, z_{1} \in W_{S_{1}}^{+}\left(A_{1}\right), z_{2} \in W_{S_{2}}^{+}\left(A_{2}\right)$;

(4) $z=z_{1}+z_{2}$ for some $z_{1} \in W_{S_{1}}^{0}\left(A_{1}\right), z_{2} \in W_{S_{2}}^{+}\left(A_{2}\right)$;

(5) $z=-\alpha z_{1}+(1-\alpha) z_{2}$ for some $0>\alpha, z_{1} \in W_{S_{1}}^{-}\left(A_{1}\right), z_{2} \in W_{S_{2}}^{+}\left(A_{2}\right)$.

We are now ready to state our result.

Proposition 2.4. Suppose $H=H_{1} \oplus H_{2}$ is a direct sum decomposition compatible with $S$ and $A$ so that $A=A_{1} \oplus A_{2}$ and $S=S_{1} \oplus S_{2}$, with $A_{j}, S_{j}$ acting on $H_{j}$ for $j=1,2$. Then

$$
W_{S}^{+}(A)=T_{1} \cup T_{2} \cup T_{3} \cup T_{4} \cup T_{5},
$$

where $T_{j}$ is the set of $z$ satisfying condition $(j)$ above for $j=1, \ldots, 5$.

Proof. Let $x=x_{1}+x_{2}, x_{1} \in H_{1}, x_{2} \in H_{2}$ be such that $(S x, x)=\left(S_{1} x_{1}, x_{1}\right)+$ $\left(S_{2} x_{2}, x_{2}\right)=1$. Then $(S A x, x)=\left(S_{1} A_{1} x_{1}, x_{1}\right)+\left(S_{2} A_{2} x_{2}, x_{2}\right)$. If $\left(S_{1} x_{1}, x_{1}\right)=0$, we have $z_{1}=\left(S_{1} A_{1} x_{1}, x_{1}\right) \in W_{S_{1}}^{0}\left(A_{1}\right)$ and $\left(S_{2} A_{2} x_{2}, x_{2}\right) \in W_{S_{2}}^{+}\left(A_{2}\right)$, and hence $(S A x, x) \in T_{4}$. Similarly, if $\left(S_{2} x_{2}, x_{2}\right)=0$, then one can show that $(S A x, x) \in T_{2}$. 
For all other cases, one can let $\alpha=\left(S_{1} x_{1}, x_{1}\right)$ and $(1-\alpha)=\left(S_{2} x_{2}, x_{2}\right)$ and show that $(S A x, x)$ is in one of the forms (1), (3) or (5).

It is easy to show that complex numbers of the form $(1)-(5)$ lie in $W_{S}(A)$. The result follows.

We have the following corollary that covers the classical result.

Corollary 2.5. Suppose $H=H_{1} \oplus H_{2}$ is a direct sum decomposition compatible with $S$ and $A$ so that $A=A_{1} \oplus A_{2}$ and $S=S_{1} \oplus S_{2}$, with $A_{j}, S_{j}$ acting on $H_{j}$ for $j=1,2$.

(a) If $S_{1}$ is positive definite, then

$$
W_{S}^{+}(A)=T_{1} \cup T_{2} \cup T_{3} .
$$

(b) If both $S_{1}$ and $S_{2}$ are positive definite, then

$$
W_{S}^{+}(A)=\operatorname{conv}\left\{W_{S_{1}}^{+}\left(A_{1}\right) \cup W_{S_{2}}^{+}\left(A_{2}\right)\right\} .
$$

\section{SPECIAL BOUNDARY POINTS AND EIGENVALUES}

As before, let $S=S^{*}, A \in L(H)$. It is well-known (e.g., see [HJ, Section 1.6]) that if $W(A)$ has some special boundary points, then $H$ is an orthogonal sum of two $A$-invariant subspaces. We obtain similar results for $W_{S}^{+}(A)$ in this section.

Theorem 3.1. Assume that $z_{0} \in W_{S}^{+}(A)$ is an angle point of $W_{S}^{+}(A)$, i.e., there exist $\theta \in \mathbf{R}$ and $\epsilon>0$ such that for every $z \in W_{S}^{+}(A)$ the inequality

$$
\operatorname{Re}\left(z-z_{0}\right) \cos \theta+\operatorname{Im}\left(z-z_{0}\right) \sin \theta \geq \epsilon\left\|z-z_{0}\right\|
$$

holds. Then for every $x_{0} \in H$ such that $\left(S x_{0}, x_{0}\right)=1$ and $z_{0}=\left(S A x_{0}, x_{0}\right)$ we have

$$
S A x_{0}=S\left(z_{0} x_{0}\right)
$$

in particular, if Ker $S=\{0\}$, then $x_{0}$ is an eigenvector of $A$ corresponding to the eigenvalue $z_{0}$. If, in addition, $S$ is invertible, then $x_{0}$ is also an eigenvector of $A^{[*]}$ corresponding to $\bar{z}_{0}$.

Here $A^{[*]}=S^{-1} A^{*} S$ is the adjoint of $A$ in the indefinite scalar product induced by $S$.

Proof. Using Proposition 2.2(a), we can assume without loss of generality that $z_{0}=0$ and $\theta=0$. The inequality (3.1) takes the form

$$
\text { Re } z \geq \epsilon\|z\|
$$

for every $z \in W_{S}^{+}(A)$. Let $x_{0} \in H$ be such that

$$
\left(S A x_{0}, x_{0}\right)=0 ; \quad\left(S x_{0}, x_{0}\right)=1 .
$$

Fix $w \in H$, and consider the vector $y=x_{0}+t w$ for $t \in \mathbf{R}$ close to zero. Let

$$
F(t)=\frac{(S A y, y)}{(S y, y)} \in W_{S}^{+}(A)
$$

We have

$$
F(t)=\frac{\left(S A\left(x_{0}+t w\right), x_{0}+t w\right)}{\left(S\left(x_{0}+t w\right), x_{0}+t w\right)}=\frac{t\left(\left(S A x_{0}, w\right)+\left(S A w, x_{0}\right)\right)+t^{2}(S A w, w)}{1+\alpha},
$$

where

$$
\alpha=t\left(\left(S x_{0}, w\right)+\left(S w, x_{0}\right)\right)+t^{2}(S w, w) .
$$


So

$$
\begin{aligned}
F(t)= & {\left[t\left(\left(S A x_{0}, w\right)+\left(S A w, x_{0}\right)\right)+t^{2}(S A w, w)\right] \cdot \frac{1}{1+\alpha} } \\
= & {\left[t\left(\left(S A x_{0}, w\right)+\left(S A w, x_{0}\right)\right)+t^{2}(S A w, w)\right] \cdot\left(1-\alpha+\alpha^{2}-\alpha^{3}+\cdots\right) } \\
= & {\left[t\left(\left(S A x_{0}, w\right)+\left(S A w, x_{0}\right)\right)+t^{2}((S A w, w)\right.} \\
& \left.\left.-\left(\left(S A x_{0}, w\right)+\left(S A w, x_{0}\right)\right)\left(\left(S x_{0}, w\right)+\left(S w, x_{0}\right)\right)\right)+\mathrm{O}\left(t^{3}\right)\right]
\end{aligned}
$$

If for some $w \in H$ we had $\operatorname{Re}\left(\left(S A x_{0}, w\right)+\left(S A w, x_{0}\right)\right) \neq 0$, then taking $t$ close to zero but of opposite signs, we would obtain a contradiction with (3.2). Thus,

$$
\operatorname{Re}\left(\left(S A x_{0}, w\right)+\left(S A w, x_{0}\right)\right)=0
$$

for all $w \in H$.

If for some $w \in H$ we had

$$
\operatorname{Im}\left(\left(S A x_{0}, w\right)+\left(S A w, x_{0}\right)\right) \neq 0,
$$

then the formula (3.3) gives

$$
\frac{\operatorname{Re} F(t)}{\operatorname{Im} F(t)} \rightarrow 0 \quad \text { as } t \rightarrow 0,
$$

a contradiction with (3.2). Thus,

$$
\operatorname{Im}\left(\left(S A x_{0}, w\right)+\left(S A w, x_{0}\right)\right)=0,
$$

for all $w \in H$. So

$$
\left(S A x_{0}, w\right)+\left(S A w, x_{0}\right)=0
$$

for all $w \in \mathbf{C}^{n}$. Replacing in (3.5) $w$ by $i w$, and comparing with (3.5), we obtain $\left(S A x_{0}, w\right)=0$ and $\left(S A w, x_{0}\right)=0$ for all $w \in H$, which implies

$$
S A x_{0}=0 ; \quad A^{*} S x_{0}=0 .
$$

If, in addition, $S$ is invertible, then the second equality in (3.6) implies $A^{[*]} x_{0}=$ 0 .

From now we assume that $S$ is invertible. Then $H$ with the indefinite scalar product induced by $S$ is a Krein space. For basic properties of geometry and operators in Krein spaces, see, e.g., [AI]. In particular, the $S$-adjoint $A^{[*]} \in L(H)$ is defined for every operator $A \in L(H)$. A (closed) subspace $\mathcal{M} \subseteq H$ is called $A$-reducing if $A x \in \mathcal{M}$ and $A^{[*]} x \in \mathcal{M}$ for every $x \in \mathcal{M}$. A subspace $\mathcal{M} \subseteq H$ is called $S$-regular if $P_{\mathcal{M}} S P_{\mathcal{M}} \in L(\mathcal{M})$ is invertible, where $P_{\mathcal{M}}$ is the orthogonal projection on $\mathcal{M}$.

Proposition 3.2. Let $\mathcal{M}$ be an $S$-regular subspace. Then the $S$-orthogonal companion

$$
\mathcal{M}^{\perp}=\{y \in H:(S y, x)=0 \text { for all } x \in \mathcal{M}\}
$$

is a direct complement of $\mathcal{M}$, and $\mathcal{M}^{\perp}$ is $S$-regular as well. If, in addition, $\mathcal{M}$ is A-reducing, then $\mathcal{M}^{\perp}$ is in turn A-reducing.

For the proof, see [GLR] (in the finite-dimensional case) or [AI] (Theorem 7.16). 
Theorem 3.3. If $z_{0}$ is an eigenvalue of $A$ having a positive eigenvector $x_{0}$, and if $z_{0}$ is a boundary point of $W_{S}^{+}(A)$, then $\operatorname{Span}\left\{x_{0}\right\}$ is an A-reducing subspace.

Proof. We may assume $\left(S x_{0}, x_{0}\right)=1$. Also, without loss of generality we may assume that $z_{0}=0$ and that $\operatorname{Re} u \geq 0$ for all $u \in W_{S}^{+}(A)$. Now we repeat the construction of the proof of Theorem 3.1. Take $w \in H$, let $y=x_{0}+t w$, and let

$$
F(t)=\frac{(S A y, y)}{(S y, y)}=\frac{t\left(S A w, x_{0}\right)+t^{2}(S A w, w)}{1+\alpha},
$$

where $\alpha=t\left(\left(S x_{0}, x\right)+\left(S w, x_{0}\right)\right)+t^{2}(S w, w)$. As in the proof of Theorem 3.1, we obtain $\operatorname{Re}\left(S A w, x_{0}\right)=0$ for all $w \in H$. Replacing $w$ by $i w$, we also obtain $\operatorname{Im}\left(S A w, x_{0}\right)=0$, hence $\left(S A w, x_{0}\right)=0$. Thus, $A^{*} S x_{0}=0$, which is equivalent to $A^{[*]} x_{0}=0$.

Corollary 3.4. Assume the hypotheses of Theorem 3.1. Let $\mathcal{M}_{+}\left(z_{0}\right)$ be a maximal $S$-positive subspace generated by vectors $x \neq 0$ such that $z_{0}=\frac{(S A x, x)}{(S x, x)}$. Assume that $\mathcal{M}_{+}\left(z_{0}\right)$ is $S$-regular. Then $\mathcal{M}_{+}\left(z_{0}\right)$ is an A-reducing subspace, and

$$
z_{0} \notin W_{S}^{+}\left(A \mid \mathcal{M}_{+}\left(z_{0}\right)^{\perp}\right) \text {. }
$$

Proof. Except for (3.7), all parts of the corollary follow from Theorem 3.1. If (3.7) were not true, then $z_{0}$ would be an angle point of $W_{S}^{+}\left(A \mid \mathcal{M}_{+}\left(z_{0}\right)^{\perp}\right)$ (because of Proposition 2.2(b)), and an application of Theorem 3.1 would produce a vector $x_{0} \in \mathcal{M}_{+}\left(z_{0}\right)^{\perp}$ such that $A x_{0}=z_{0} x_{0}$ and $\left(S x_{0}, x_{0}\right)=1$. Then $\mathcal{M}:=\mathcal{M}_{+}\left(z_{0}\right)+$ $\operatorname{Span}\left\{x_{0}\right\}$ would be an $S$-positive subspace generated by the vectors $x \neq 0$ such that $\frac{(S A x, x)}{(S x, x)}=z_{0}$. But $\mathcal{M}$ is larger than $\mathcal{M}_{+}\left(z_{0}\right)$, a contradiction with the maximality of $\mathcal{M}_{+}\left(z_{0}\right)$.

Note that in the finite-dimensional case every $S$-positive subspace is $S$-regular. This is generally false in infinite-dimensional Krein spaces.

\section{Matrices having polygonal numerical Range}

We continue to assume in this section that $S$ is nonsingular. In addition, it will be assumed in this section that $H$ is finite dimensional.

We say that $W_{S}^{+}(A)$ is polygonal if $W_{S}^{+}(A)$ is the intersection of a finite number of closed half-planes.

In the definite case ( $S$ positive definite, and we may assume $S=I$ without essential loss of generality), the answer is well known (see, for example, section 3.10 in [GL], or section 1.6 in [HJ]): $W_{S}^{+}(A)$ is polygonal if and only if there is an $A$ reducing subspace $\mathcal{M}$ such that $A \mid \mathcal{M}$ is normal with eigenvalues at the vertices of $W_{S}^{+}(A)$, and $W_{S}^{+}\left(A \mid \mathcal{M}^{\perp}\right)$ is contained in $W_{S}^{+}(A)$ but does not contain any vertices of $W_{S}^{+}(A)$.

In the indefinite case the situation is more complicated because $W_{S}^{+}(A)$ is often unbounded (see Theorem 2.3).

Consider first the case when $W_{S}^{+}(A)$ has no angle points.

Theorem 4.1. Assume that $W_{S}^{+}(A)$ is polygonal and has no angle points. Then only the following cases can occur:

(a) $W_{S}^{+}(A)$ is a straight line. This happens if and only if $A$ has the form $A=$ $\alpha I+\beta B$, where $\alpha, \beta \in \mathbf{C}$, and $B$ is $S$-self-adjoint such that $S B$ is indefinite; 
(b) $W_{S}^{+}(A)$ coincides with $\mathbf{C}$;

(c) $W_{S}^{+}(A)$ is a closed half-plane.

Proof. Observe that under the hypotheses of the theorem the set $W_{S}^{+}(A)$ is unbounded. We show first of all that the case when $W_{S}^{+}(A)$ is the strip between two parallel lines is impossible. Assume the contrary. Using Proposition 2.2(a), we can assume without loss of generality that

$$
W_{S}^{+}(A)=\left\{(x, y) \in \mathbf{R}^{2}:-1 \leq x \leq 1\right\} .
$$

By Proposition 2.1,

$$
W_{S}^{+}(A)=K\left(G_{1}, G_{2}, S\right) \cap\{(x, y, 1): x, y \in \mathbf{R}\},
$$

where

$$
K\left(G_{1}, G_{2}, S\right)=\left\{\left(u^{*} G_{1} u, u^{*} G_{2} u, u^{*} S u\right): u \in \mathbf{C}^{n}\right\},
$$

and the Hermitian matrices $G_{1}, G_{2}$ are such that $S A=G_{1}+i G_{2}$. Since $K\left(G_{1}, G_{2}, S\right)$ is a closed convex cone (see, e.g., [AT]), we must have that $K\left(G_{1}, G_{2}, S\right)$ coincides with the wedge $\{(x, y, z) \in \mathbf{R}: z \geq 0,|x| \leq z\}$. In particular, $S \geq 0$. But $S$ is invertible, so, in fact, $S$ is positive definite, a contradiction with $W_{S}^{+}(A)$ being an unbounded set.

Now clearly one of (a), (b), or (c) holds. It follows from [BL] that $W_{S}^{+}(A)$ is a straight line if and only if the condition indicated in (a) holds.

Remark 4.2. Note that Theorem 4.1 actually holds for singular $S$ as well. One can use the same arguments in the first paragraph of our proof until we arrive at the conclusion that $S \geq 0$. At this point, if $S$ is singular, we can find a unitary matrix $U$ such that $U^{*} S U=S_{1} \oplus 0$, where $S_{1}$ is positive definite. Then $U^{*}\left(G_{1}+i G_{2}\right) U=$ $\left(U^{*} S U\right)\left(U^{*} A U\right)$ is of the form $\left(\begin{array}{cc}B_{1} & B_{2} \\ 0 & 0\end{array}\right)$, where $B_{1}$ has the same size as $S_{1}$. By Theorem 2.3 in [LTU], if $B_{2} \neq 0$ then $W_{S}^{+}(A)=\mathbf{C}$; if $B_{2}=0$ then $W_{S}^{+}(A)$ is bounded. Both cases contradict the assumption that $W_{S}^{+}(A)$ is a strip between two parallel lines. One can then complete the proof as before.

Consider now the case when $W_{S}^{+}(A)$ has angle points.

Theorem 4.3. Let $z_{1}, \ldots, z_{k}$ be the angle points of $W_{S}^{+}(A)$. Then there exist mutually $S$-orthogonal A-reducing subspaces $\mathcal{M}_{1}, \ldots, \mathcal{M}_{k+1}$ with the following properties:

(i) $\mathcal{M}_{1}, \ldots, \mathcal{M}_{k}$ are $S$-positive;

(ii) $A x=z_{j} x$ for every $x \in \mathcal{M}_{j}(j=1, \ldots, k)$;

(iii) $z_{1}, \ldots, z_{k}$ do not belong to $W_{S}^{+}\left(A \mid \mathcal{M}_{k+1}\right)$.

Proof. Let $\mathcal{M}_{1}$ be a maximal $S$-positive subspace generated by vectors $x \neq 0$ such that $z_{1}=\frac{(S A x, x)}{(S x, x)}$. By Theorem 3.1, $A x=z x$ for every $x \in \mathcal{M}_{1}$. By Corollary 3.4, $\mathcal{M}_{1}$ is $A$-reducing, and $z_{1} \notin W_{P_{1} S P_{1}}^{+}\left(A \mid \mathcal{M}_{1}^{\perp}\right)$, where $P_{1}$ is the orthogonal projection on $\mathcal{M}_{1}^{\perp}$. By Proposition 2.2(b),

$$
W_{P_{1} S P_{1}}^{+}\left(A \mid \mathcal{M}_{1}^{\perp}\right) \subseteq W_{S}^{+}(A) .
$$

On the other hand, we claim that $z_{2}, \ldots, z_{k}$ are angle points of $W_{P_{1} S P_{1}}^{+}\left(A \mid \mathcal{M}_{1}^{\perp}\right)$. Indeed, in view of (4.1) we only have to prove that $z_{2}, \ldots, z_{k} \in W_{P_{1} S P_{1}}^{+}\left(A \mid \mathcal{M}_{1}^{\perp}\right)$. 
Consider $z_{2}$. By Proposition 2.4 we have (in view of $W_{\left(I-P_{1}\right) S\left(I-P_{1}\right)}^{+}\left(A \mid \mathcal{M}_{1}\right)=$ $\left.\left\{z_{1}\right\}\right)$

$$
\begin{aligned}
W_{S}^{+}(A)= & \bigcup_{0 \leq \alpha<1}\left\{\alpha\left\{z_{1}\right\}+(1-\alpha) W_{P_{1} S P_{1}}^{+}\left(A \mid \mathcal{M}_{1}^{\perp}\right)\right\} \cup \\
& \bigcup_{\alpha>1}\left\{\alpha\left\{z_{1}\right\}+(1-\alpha) W_{P_{1} S P_{1}}^{-}\left(A \mid \mathcal{M}_{1}^{\perp}\right)\right\} \cup\left\{\left\{z_{1}\right\}+W_{P_{1} S P_{1}}^{0}\left(A \mid \mathcal{M}_{1}^{\perp}\right)\right\} .
\end{aligned}
$$

According to (4.2), three cases (which are not mutually exclusive) can occur:

$$
\begin{aligned}
& z_{2}=\alpha z_{1}+(1-\alpha) u, \quad u \in W_{P_{1} S P_{1}}^{+}\left(A \mid \mathcal{M}_{1}^{\perp}\right), \quad 0 \leq \alpha<1 \\
& z_{2}=\alpha z_{1}+(1-\alpha) v, \quad v \in W_{P_{1} S P_{1}}^{-}\left(A \mid \mathcal{M}_{1}^{\perp}\right), \quad \alpha>1 ; \\
& z_{2}=z_{1}+w, \quad w \in W_{P_{1} S P_{1}}^{0}\left(A \mid \mathcal{M}_{1}^{\perp}\right) .
\end{aligned}
$$

If (4.3) holds, then clearly $u=z_{2}$ and $\alpha=0$ (otherwise $z_{2}$ would not be an angle point of $W_{S}^{+}(A)$ ). If (4.4) holds, then by (4.2) the whole ray $\left\{\alpha z_{1}+(1-\alpha) v: \alpha \geq 1\right\}$ belongs to $W_{S}^{+}(A)$, again a contradiction with $z_{2}$ being an angle point of $W_{S}^{+}(A)$. If (4.5) holds, then (since $W_{P_{1} S P_{1}}^{0}\left(A \mid \mathcal{M}_{1}^{\perp}\right)$ is a cone) in view of (4.2) the whole ray $\left\{z_{1}+\alpha w: \alpha>0\right\}$ belongs to $W_{S}^{+}(A)$, a contradiction again. It follows therefore that $z_{2} \in W_{P_{1} S P_{1}}^{+}\left(A \mid \mathcal{M}_{1}^{\perp}\right)$, as claimed.

The proof of Theorem 4.3 is now easily completed using induction on $k$, and applying the induction hypothesis to $P_{1} S P_{1}$ in place of $S$ and to $A \mid \mathcal{M}_{1}^{\perp}$ in place of $A$.

In contrast with the classical numerical range result, one cannot claim in Theorem 4.3 that $W_{S}^{+}\left(A \mid \mathcal{M}_{k+1}\right)$ is always contained in $W_{S}^{+}(A \mid \mathcal{M})$, where $\mathcal{M}=\mathcal{M}_{1}+$ $\cdots+\mathcal{M}_{k}$. Indeed, since $\mathcal{M}$ is $S$-positive, the numerical range $W_{S}^{+}(A \mid \mathcal{M})$ is bounded, while $W_{S}^{+}\left(A \mid \mathcal{M}_{k+1}\right)$ need not be bounded. Using Theorem 2.3 it is easy to check that if $W_{S}^{+}(A)$ is bounded, then (under the hypotheses of Theorem 4.3) the containment $W_{S}^{+}\left(A \mid \mathcal{M}_{k+1}\right) \subseteq W_{S}^{+}(A \mid \mathcal{M})$ holds true.

\section{ACKNOWLEDGMENTS}

We thank V. Strauss for several helpful discussions concerning numerical ranges of operators in finite dimensional Krein spaces. We also thank C.K. Sze for letting us know about his Master's thesis [S] in which the geometry of the set $W_{S}^{+}(A) \cup$ $W_{S}^{-}(A)$ is analysed in the context of finite dimensional indefinite product spaces, in particular in connection with the class of operators that are normal in the indefinite scalar product.

\section{REFERENCES}

[AT] Y.H. Au-Yeung and N.K. Tsing, An extension of the Hausdorff-Toeplitz theorem on the numerical range, Proc. Amer. Math. Soc. 89 (1983), 215-218. MR 85f:15021

[AI] T. Ya. Azizov and I. Iohvidov, Linear Operators in Spaces with Indefinite Metric, J. Wiley and Sons, 1989, (Translation from Russian). MR 90j:47042

[B] T. Bayasgalan, The numerical range of linear operators in spaces with an indefinite metric (Russian), Acta Math. Hungar. 57 (1991), 7-9. MR 93a:47036

[BL] P. Binding and C.K. Li, Joint ranges of Hermitian matrices and simultaneous diagonalization, Linear Algebra Appl. 151 (1991), 157-168. MR 92d:47006 
[D] R.G. Douglas, On majorization, factorization, and range inclusion of operators in Hilbert space, Proc. Amer. Math. Soc. 17 (1966), 413-415. MR 34:3315

[GL] I.M. Glazman and Yu. I. Lyubich, Finite Dimensional Linear Analysis, Nauka, Moscow, 1969 (in Russian; English transl., M.I.T. Press, Cambridge, MA, 1974). MR 50:7192; MR 50:7195

[GLR] I. Gohberg, P. Lancaster and L. Rodman, Matrices and Indefinite Scalar Products, Birkhäuser, 1983. MR 87j:15001

[GP] R.D. Grigorieff and R. Plato, On the minimax equality for seminorms, Linear Algebra Appl. 221 (1995), 227-243. MR 96e:15049

[HJ] R.A. Horn and C.R. Johnson, Topics in Matrix Analysis, Cambridge University Press, Cambridge, 1991. MR 92e:15003

[LTU] C.K.Li, N.K.Tsing and F. Uhlig, Numerical ranges of an operator on an indefinite inner product space, Electronic Linear Algebra 1 (1996), 1-17. MR 97g:47005

[S] C.K. Sze, S-normality and polygonal S-numerical ranges, M. Phil. Thesis, University of Hong Kong, 1997.

Department of Mathematics, The College of William and Mary, Williamsburg, VirGINIA 23187

E-mail address: ckli@math.wm.edu

E-mail address: lxrodm@math.wm.edu 